\title{
Diversité et usages des plantes médicinales à l'ouest du Niger
}

\author{
Abdou Laouali*1, Moussa Mamoudou Boubacar ${ }^{1}$, Baggnian Issoufou ${ }^{2}$, Mahamane Ali ${ }^{1,3}$ \\ 1 Université de Diffa, Faculté des Sciences Agronomiques, BP 78, Diffa, Niger; \\ 2 Département des Ressources Naturelles et de l'Environnement, Faculté des Sciences Agronomiques (FSA), Université de \\ Tahoua, Niger \\ 3 Université Abdou Moumouni, Faculté des Sciences et Techniques, Département de Biologie, Laboratoire Garba Mounkaila, \\ BP 10662 Niamey-Niger \\ *Auteur correspondant : abdoulaouali2000@yahoo.fr
}

Mots clés : Plantes médicinales, Pharmacopée, Tradipraticiens, Niger

Keywords : Medicinal plants, Pharmacopoeia, Traditional practitioner, Niger

Publication date 30/11/2020, http://m.elewa.org/Journals/about-japs/

\section{RESUME}

La médecine traditionnelle est profondément ancrée dans la culture de nombreux pays d'Afrique de l'ouest. L'objectif du présent travail était de contribuer à la connaissance des plantes médicinales et de leurs utilisations dans le département de Dogondoutchi à l'ouest du Niger. Un échantillon de (19) villages a été retenu dans deux communes du département. Les données ont été collectées à travers un guide d'entretien adressé à 49 personnes appartenant à différents groupes socioprofessionnels, à raison de deux à trois par village. Ces entretiens ont permis d'inventorier 55 espèces médicinales réparties en 26 familles. Les familles les plus représentées sont les Fabaceae-Caesalpinoideae (10 espèces) suivies des Fabaceae-Mimosoideae ( 7 espèces) et des Combretaceae ( 5 espèces). Les maladies couramment traitées sont les hémorroïdes, le paludisme, la fièvre et la dysenterie. La décoction, la poudre, la macération, l'infusion et la calcination constituent les modes de préparation. Les parties utilisées sont les feuilles, les écorces, les racines, les graines, les fruits les plantes entières (herbacées) et les rameaux. Beaucoup de plantes médicinales se raréfient ou ont déjà disparu dans la zone d'étude. L'adoption de méthodes de gestion durable s'avère nécessaire pour sauvegarder les plantes médicinales dans cette zone.

\begin{abstract}
Traditional medicine is deeply rooted in the culture of many countries in West Africa. The objective of this work was to contribute to the knowledge of medicinal plants and their uses in the department of Dogondoutchi in western Niger. A sample of (19) villages was selected in two communes of the department. The data was collected through an interview guide addressed to 49 people belonging to different socio-professional groups, two to three per village. These interviews allowed to inventory 55 medicinal species divided into 26 families. The most represented families are Fabaceae-Caesalpinoideae (10 species) followed by Fabaceae-Mimosoideae (7 species) and Combretaceae (5 species). Commonly treated diseases are hemorrhoids, malaria, fever, and dysentery. decoction, powder, maceration, infusion and calcination are the methods of preparation. The parts used are leaves, bark, roots, seeds, fruits, whole plants (herbaceous) and twigs. Many medicinal plants are becoming scarce or have already disappeared in the study area. There is an urgent need to adopt a sustainable management methods to save medicinal plants in this area.
\end{abstract}




\section{INTRODUCTION}

Après la conférence de Rio en 1992, la question la plus importante dans le domaine des connaissances traditionnelles était de savoir comment certains groupes ethniques ou la population locale utilisent et conservent leur environnement. En Afrique, les plantes médicinales constituent des ressources précieuses pour la majorité des populations rurales et urbaines et sont les principaux moyens par lesquels les individus se soignent (Badiaga, 2011). En milieu rural, malgré l'avènement des produits pharmaceutiques modernes, ceux-ci restent inaccessibles aux pauvres populations. Ceci les oblige à continuer à se tourner vers les plantes pour leurs soins médicaux. Pour pérenniser ces connaissances ancestrales, plusieurs études ont été menées: (Koné et al. (2002) en Côte d'Ivoire, Togola et al. (2005) au Mali, Abdou et al. (2014), Baggnian et al. (2018), Garba et al. (2019) et Abdou Habou et al., (2020) au Niger. On assiste au cours de ces dernières décennies à un amenuisement des ressources biologiques en général et végétales en particulier dans leur répartition spatiale ainsi que leur diversité biologique. En effet plusieurs espèces ont disparu ou sont en voie de disparition de leur habitat naturel à cause du changement climatique et de la pression anthropique croissante. La connaissance précise des plantes utilisées par chaque communauté et l'adoption de mesures de gestion durable au niveau local s'imposent, afin de préserver et sauvegarder la diversité biologique utilisée par les populations rurales. Ce travail s'inscrit dans ce cadre et a pour objectif général de contribuer à la connaissance des plantes médicinales et de leurs utilisations dans le département de Dogondoutchi. De façon spécifique, il s'agit d'identifier les tradipraticiens, de répertorier les plantes médicinales, d'identifier les principaux usages médicinaux de ces plantes et d'inventorier celles qui sont en voie de disparition.

MATERIEL ET METHODES

3.1 Présentation de la zone d'étude: département de Dogondoutchi, région de Dosso Cette étude a été réalisée dans les communes de (figure 1). Kieché et Soucoucoutane, localisées dans le 


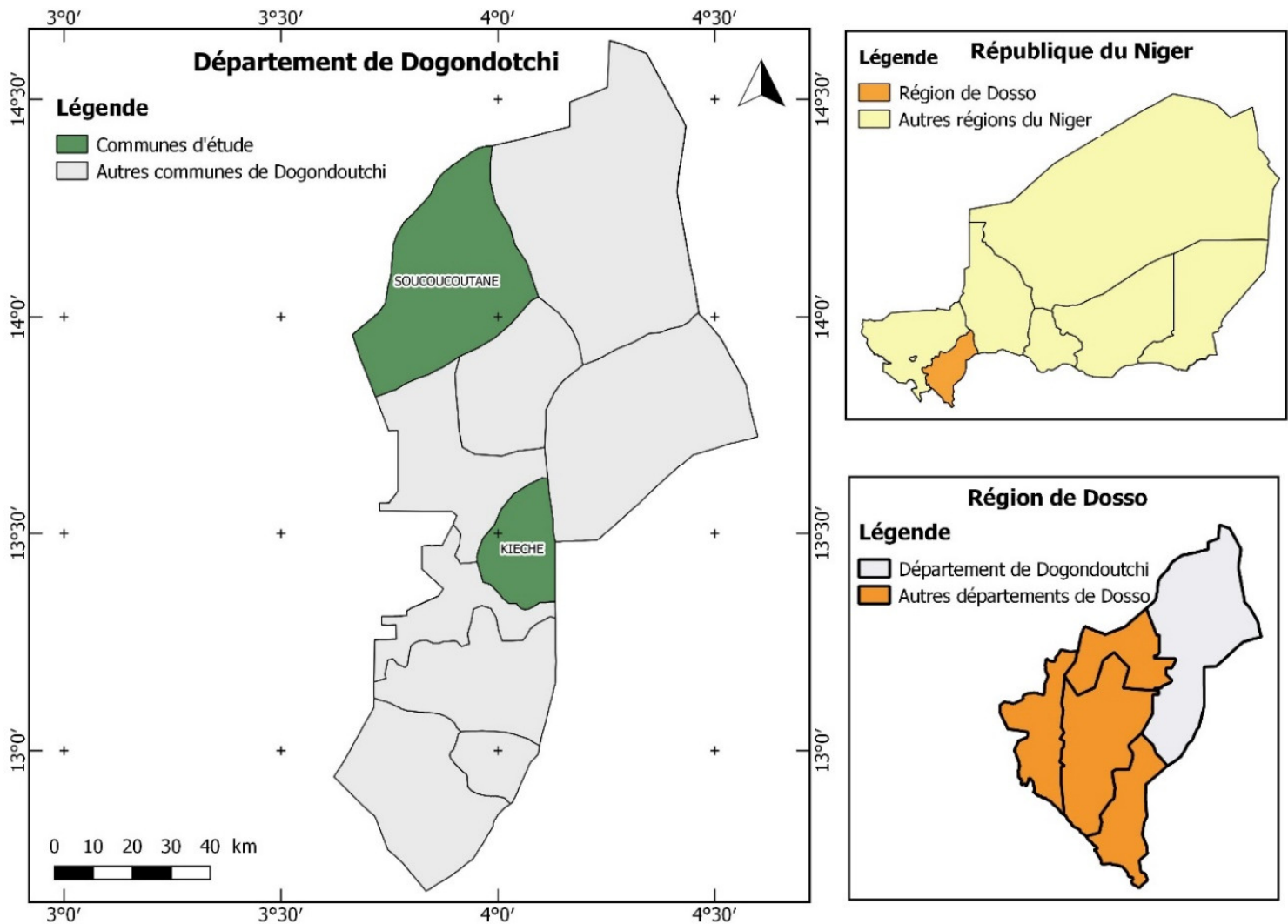

Figure 1 : Localisation de la zone d'étude

Le climat est de type sahélo soudanien dont les précipitations varient entre $550 \mathrm{~mm}$ à $600 \mathrm{~mm}$. On note une réduction substantielle de ces précipitations du sud vers le nord et d'Est en Ouest. Le régime des vents est marqué par l'harmattan qui est un vent chaud et sec qui souffle du Nord au Sud et la mousson qui est un vent humide soufflant d'Est en Ouest. Les sols sont sableux au centre et à l'ouest, ferrugineux tropicaux à l'est et alluvionnaires dans les zones dépressionnaires. La végétation est dominée par un parc Agroforestier à Faidherbia albida (Del.) A. Chev. dans la vallée du dallol, des combrétaceae (Combretum micranthum, Combretum nigricans) et des aires de pâturage constituées de Guiera senegalensis, Combretum glutinosum, Annona senegalensis, Balanites aegyptiaca sur les plateaux. La flore herbacée est composée principalement de : Cenchrus biflorus, Eragrostis sp, Andropogon gayanus, Andropogon Giganteus (CUD, 2017). La population du département de Dogondoutchi est estimée à 372473 habitants dont 183802 hommes $(49,3 \%)$ et 188671 femmes (50,7\%) (INS, 2016).
Les cultures pluviales et les cultures irriguées constituent les principales activités agricoles des populations du département de Dogondoutchi. L'élevage des espèces caprines, ovines, bovines, asines, équines, camelines, avicoles constitue la deuxième activité économique des populations.

3.2 Echantillonnage et collecte des données : Un échantillon de (19) villages a été retenu dont douze (12) dans la commune de Kieché et sept (7) dans celle de Soucoucoutane. Dans chaque village, deux à trois personnes ont été enquêtés soit au total 49 personnes, choisies sur la base des critères de connaissances en pharmacopée traditionnelle et de représentation socioprofessionnels. La collecte des données a été effectuée entre juillet et septembre 2018 à travers un questionnaire s'adressant aux différents groupes socioprofessionnels. Chaque acteur concerné a été questionné individuellement. Les informations collectées ont concerné les plantes utilisées, les différents usages des plantes (modes de préparation et d'administration des produits, maladies traitées, 
organes utilisés, mode et période de prélèvement), les plantes disparues ou en voie de disparition et les causes de leur disparition.

3.3 Traitement et analyse des données : Un test de corrélation de Pearson a été effectué pour déterminer les liens entre les variables et une analyse des correspondances multiples (ACM) pour faire ressortir les variables les mieux corrélées aux principaux axes et aussi le degré de dépendance entre les modalités des variables. Le package factoextra du logiciel $\mathrm{R}$ ( développement Coré Team, 2005) a été utilisé à cet effet. Les packages ggplot2 et ggpubr ont été aussi utilisés pour une bonne représentation des tableaux multifactoriels.

médicinales est répandue chez les tranches d'âge de 40 à 90 ans, avec une prédominance chez les personnes âgées de 60 à 70 ans (32,65\%) (figure 2).

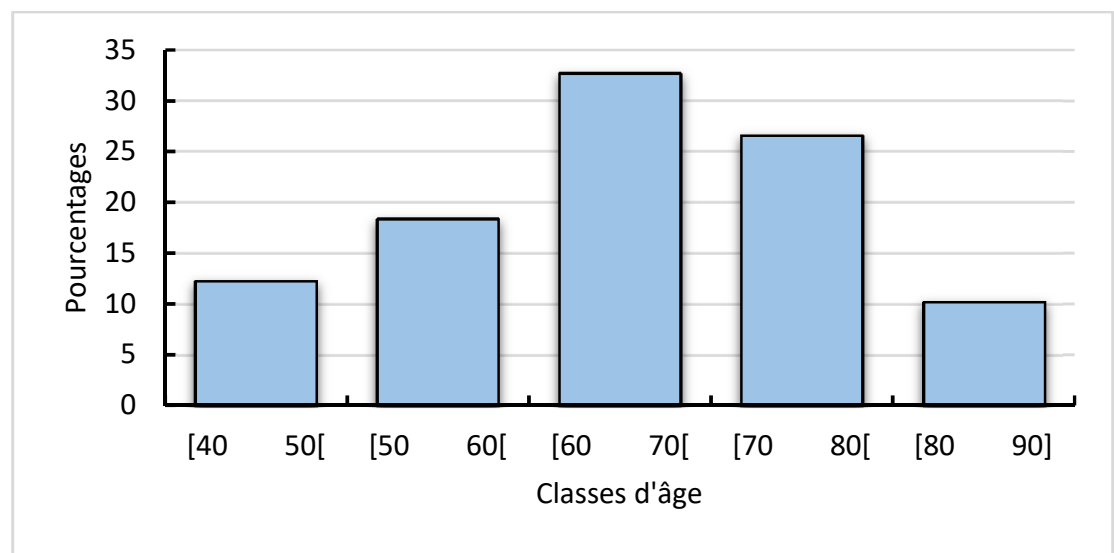

Figure 2 : Age des tradipraticiens enquêtés

Parmi ces tradipraticiens $81,63 \%$ sont ambulants et $18,37 \%$ sont fixes. Le niveau de scolarisation de la population enquêtée est faible : $12,25 \%$ ont le niveau primaire, $32,65 \%$ sont analphabètes et $55,10 \%$ ont fréquenté l'école coranique.

4.2 Composition taxonomique des plantes médicinales citées : Les entretiens ont permis d'inventorier 55 espèces médicinales réparties en 26 familles (tableau 1). Avec 10 espèces, la famille des Fabaceae-Caesalpinoieae est la plus représentée. Elle est suivie des Fabaceae-Mimosoideae (7 espèces) et Combretaceae (5 espèces). Certaines des espèces citées ont disparu dans la commune de Soucoucoutane ou sont en voie de disparition dans les deux communes. 
Tableau 1 : Plantes médicinales citées

\begin{tabular}{|c|c|c|c|}
\hline Famille & Espèces & Famille & Espèces \\
\hline Amaryllidaceae & Crinum ornatum & \multirow{7}{*}{ Fabaceae-Mimosoideae } & Acacia macrostachya* \\
\hline \multirow{3}{*}{ Anacardiaceae } & Lannea acida* & & Acacia nilotica \\
\hline & Lannea microcarpa* & & Acacia radiana \\
\hline & Sclerocarya birrea* & & Albizzia chevalieri \\
\hline Annonaceae & Annona senegalensis & & Faidherbia albida \\
\hline \multirow{2}{*}{ Apocynaceae } & Leptadenia hastata & & Parkia biglobosa \\
\hline & Calotropis procera & & Prosopis africana** \\
\hline Asclepiadaceae & Pergularia tomentosa & Lamiaceae & Vitex doniana* \\
\hline Balanitaceae & Balanites aegyptiaca & Loganiaceae & Strychnos spinosa* \\
\hline Bombacaceae & Adansonia digitata & \multirow{3}{*}{ Malvaceae } & Bombax costatum* \\
\hline Burceraceae & Boswelia odorata & & Hibiscus esculentus \\
\hline \multirow{2}{*}{ Capparaceae } & Boscia angustifolia & & Sterculia setigera* \\
\hline & Boscia senegalensis & \multirow{3}{*}{ Meliaceae } & Azadirachta indica \\
\hline \multirow{5}{*}{ Combretaceae } & Anogeissus leiocarpa & & Khaya senegalensis* \\
\hline & Combretum glutinosum* & & Pseudocedrela kostchyi \\
\hline & Combretum micranthum & Moringaceae & Moringa oleifera \\
\hline & Guiera senegalensis & Myrtaceae & Eucaliptus camaldulensis \\
\hline & Terminalia avicennioides* & Phyllantaceae & Securinega virosa \\
\hline \multirow{2}{*}{ Convolvulaceae } & Merrenia tridentata & Poaceae & Pennisetum glaucum \\
\hline & Evolvulus alsinoides & Polygalaceae & Securidaca longepedunculata** \\
\hline Ebenaceae & Diospyros mespiliformis & Rhamnaceae & Ziziphus mauritiana \\
\hline \multirow{2}{*}{ Euphorbiaceae } & Chrozophora brochiana & Sapotaceae & Vitellaria paradoxa* \\
\hline & Croton zambesicus & & \\
\hline \multirow{10}{*}{$\begin{array}{l}\text { Fabaceae- } \\
\text { Caesalpinoideae }\end{array}$} & Baubinia rufescens & & \\
\hline & Cassia italica* $^{*}$ & & \\
\hline & Cassia mimosoides & & \\
\hline & Cassia nigricans & & \\
\hline & Cassia obtusifolia & & \\
\hline & Cassia occidentalis & & \\
\hline & Cassia sieberiana & & \\
\hline & Cassia tora & & \\
\hline & Piliostigma reticulatum & & \\
\hline & Tamarindus indica** & & \\
\hline
\end{tabular}

* : Espèce en voie de disparition ; ${ }^{* *}$ : Espèce disparue dans la commune de Soucoucoutane

D'après les tradipraticiens, la disparition est due aux actions anthropiques (coupe abusive (65,30\%), manque d'entretien (20,41\%), mauvaise pratique de certaines personnes sur ces espèces $(14,29 \%))$.

4.3 Organes prélevés, modes de préparation et formes d'utilisation: Les parties ou organes prélevés pour les traitements des pathologies sont : les feuilles, les écorces, les racines, les plantes entières (herbacées), les rameaux, les graines et les fruits (figure 3). Les modalités de préparation les plus citées sont la décoction ( $82 \%$ ) et la poudre $(22,45 \%)$ (figure 4$)$. L'administration se fait par la voie orale qui est la plus fréquente, la voie cutanée et la voie nasale. 


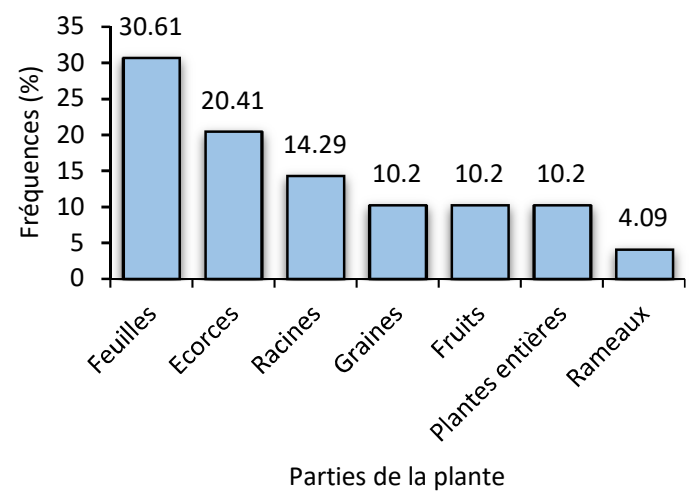

Figure 3 : Fréquence d'utilisation des différentes parties de la plante

4.4 Techniques et moment de la récolte des parties de la plante et outils utilisés : Plusieurs techniques sont utilisées pour la récolte des plantes (figure 5) et les outils utilisés sont la hache $(55,1 \%)$ et le couteau $(44,9 \%)$. Cette récolte se fait avec soin, mais malgré cela, la mauvaise utilisation des outils peut engendrer la perte de nombreuses espèces végétales dans le milieu. La majorité des personnes interrogées

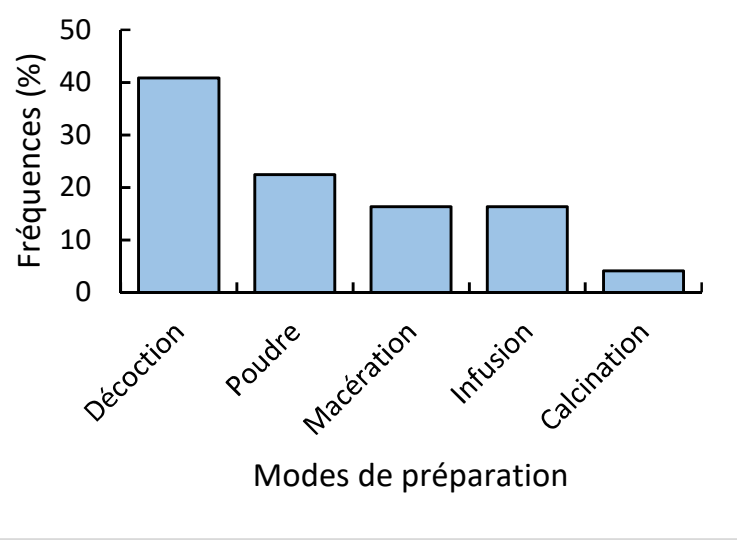

Figure 4 : Modes de préparation des médicaments

ont notifié que le prélèvement des plantes médicinales se fait pendant la saison sèche parce qu'elles ont une faible teneur en eau et sont plus efficaces (figure 6). Néanmoins, cette récolte peut se faire à tout moment en fonction du besoin. Pour les espèces herbacées, la récolte se fait surtout pendant la saison hivernale et la conservation se fait par séchage en pilant les produits ou en les laissant comme tels.

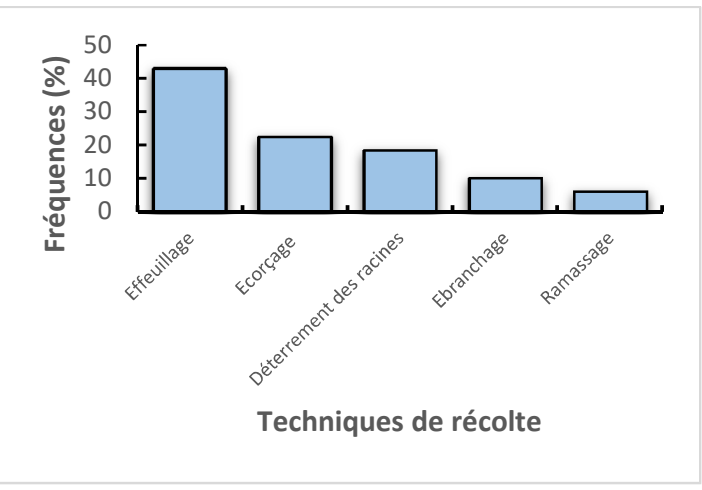

Figure 5 : Fréquences des techniques utilisées pour la récolte

4.5 Acquisition des connaissances : La connaissance des propriétés et usages des plantes est généralement acquise par expérience et transmise de génération en génération. Les modes de transmission des connaissances sont du père au fils $(85,71 \%)$ et la collaboration avec une autre personne expérimentée $(14,29 \%)$.

4.6 Liens entre les différentes variables : L'analyse des correspondances multiples

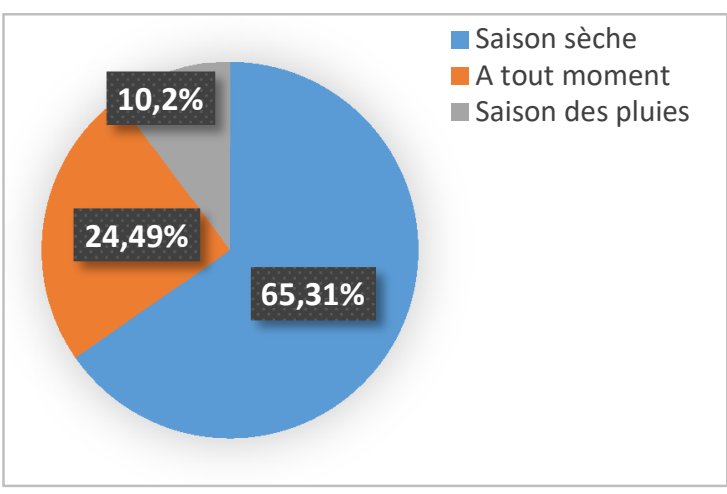

Figure 6 : Moment de la récolte

effectuée montre le degré de corrélation entre les variables et les principaux axes d'une part et le degré de dépendance entre les modalités des variables d'autre part. La figure 7 permet de distinguer trois groupes de variables selon leurs degrés de corrélation avec les deux premiers axes. Les plantes utilisées et les maladies traitées constituent le groupe (I) le plus corrélé $(r>0,8)$ suivi du groupe II constitué des techniques 


IOURNAL
OT
NNIMAL

utilisées, du mode de préparation et des organes utilisés $(0,4<\mathrm{r}<0.8)$. Le groupe III faiblement corrélé $(r<0,3)$ est formé de type biologique, de moment de récolte, de mode d'administration, des connaissances acquises et des outils utilisés. En se basant sur les groupes bien corrélés (I et II), on peut noter les dépendances entre certaines modalités selon leur degré de contribution (contrib) dans la définition des axes (Figure 8). Il s'agit principalement, suivant l'axe 1, de l'utilisation des racines de Calotropis procera pour traiter la syphilis. Suivant l'axe 2, la décoction des feuilles de Azadirachta indica pour traiter les maux de ventre et les écorces de Moringa oleifera pour traiter la dysenterie.

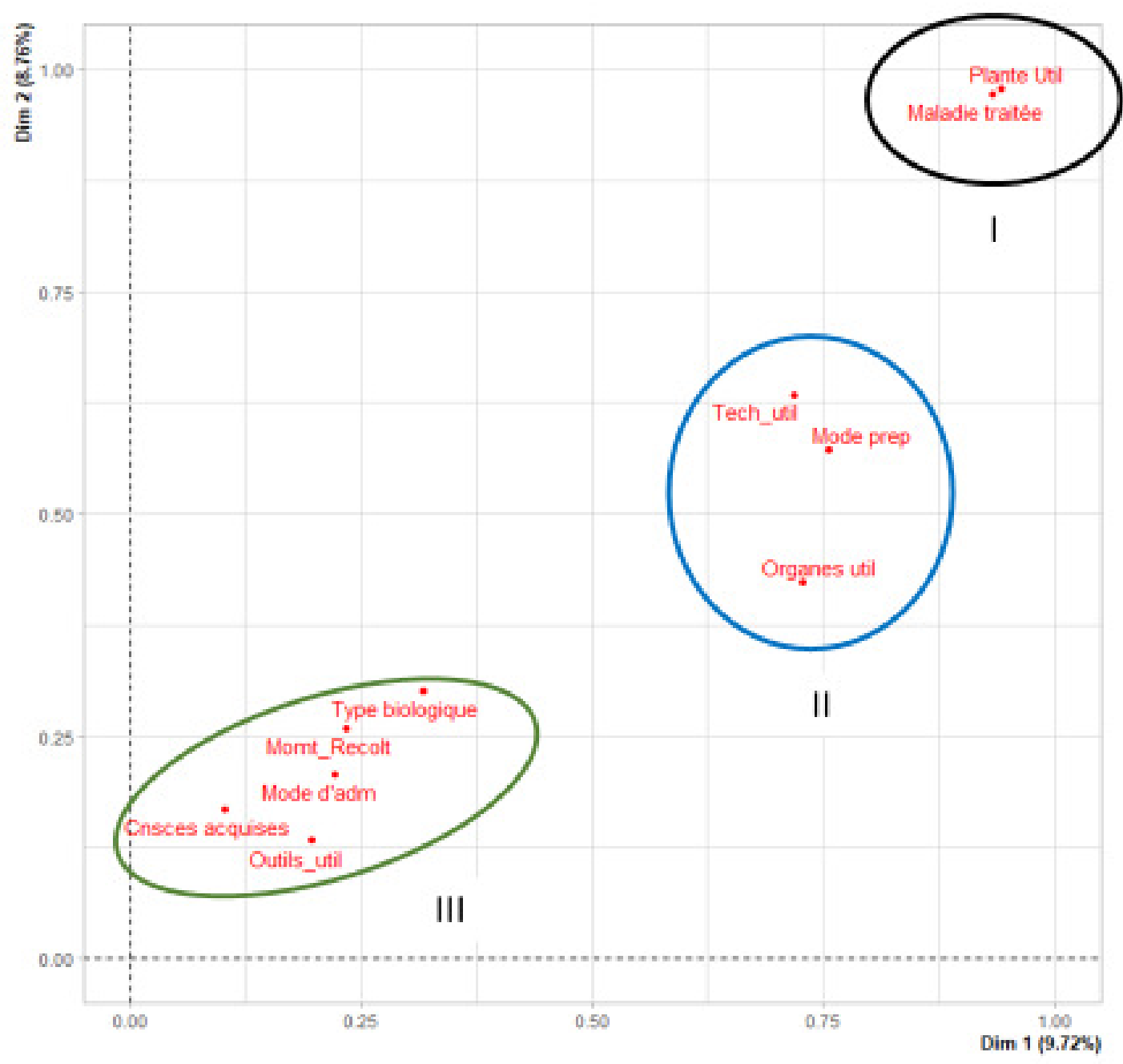

Figure 7 : Carte factorielle de la contribution des variables 

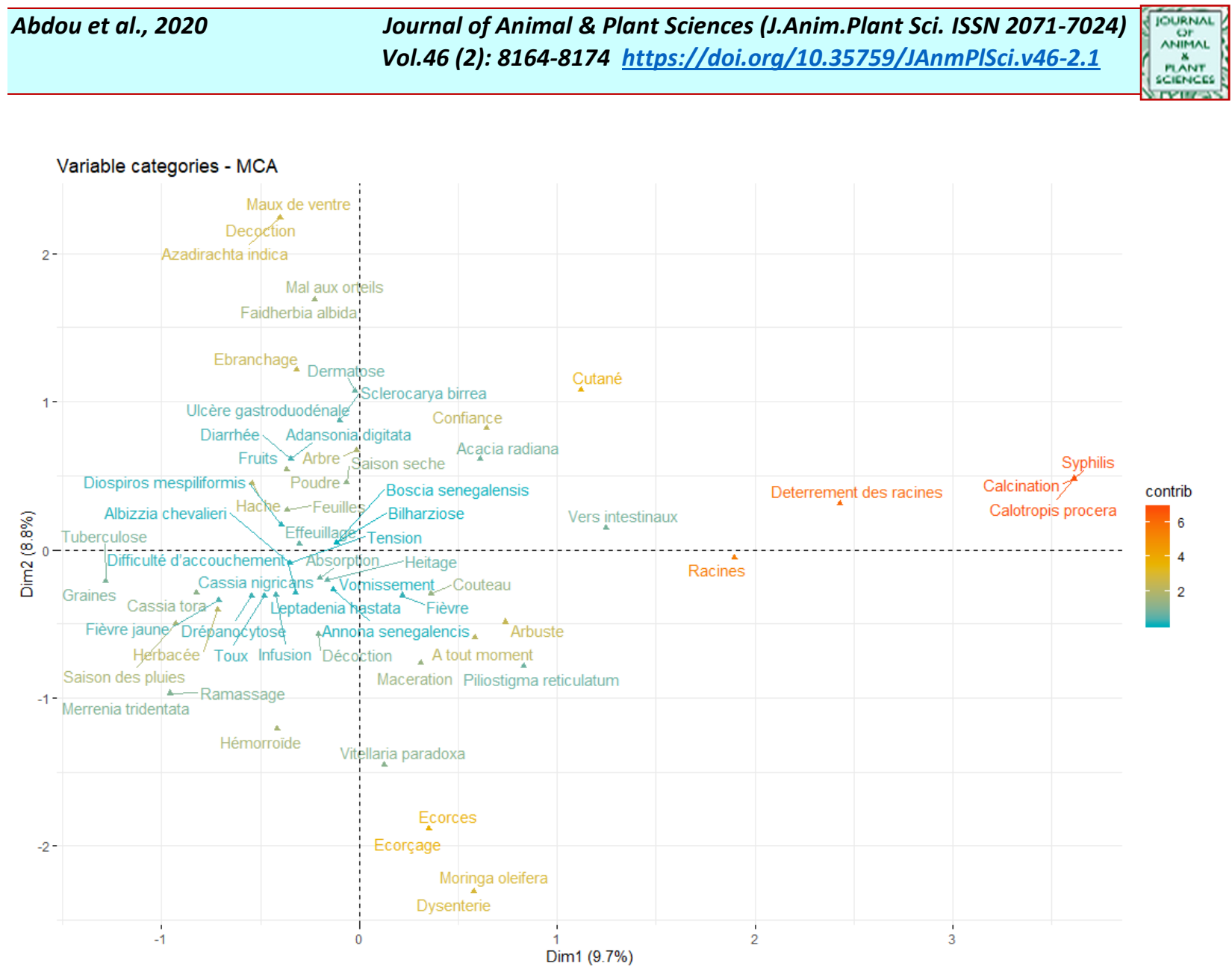

Figure 8 : Carte factorielle des catégories de variables et la contribution des catégories (contrib)

\section{DISCUSSION}

A l'issue des enquêtes, 49 personnes dont 37 hommes et 12 femmes ont été interrogées. La plupart des tradipraticiens étaient majoritairement de sexe masculin, ce qui corrobore les résultats de Sangaré et al. (2012) au Bénin concernant cette faible implication des femmes dans l'exercice de la médecine traditionnelle. Cependant, Mehdioui et Kahouadji (2007) ont trouvé au Maroc que les femmes $(53 \%)$ connaissent mieux les plantes médicinales que les hommes (47\%). Les personnes enquêtées ont une tranche d'âge comprise entre 40 et 90 ans. Le résultat de cette étude est proche de celui observé par Mehdioui et Kahouadji (2007) et de celui de Mike et al. (2014) au Sud du Nigéria avec respectivement 61 $\%$ et $78,12 \%$ d'hommes dont l'âge est égal ou supérieur à 40 ans. Plusieurs autres auteurs (Betti et Lejoly, 2000 ; Guedje et al., 2008 ; Jiofack et al., 2010 ; Mpondo et al., 2012) ont également trouvé que les connaissances traditionnelles sont souvent beaucoup plus détenues par les personnes âgées. Cette répartition des connaissances en faveur des personnes âgées pourrait causer à long terme la disparition des connaissances traditionnelles locales. Le niveau de scolarisation de la population enquêtée est faible, $12,25 \%$ seulement ont le niveau primaire, $32,65 \%$ sont analphabètes mais $55,10 \%$ ont fréquenté l'école coranique. Ces résultats corroborent ceux de Roufai (2013) qui a aussi constaté ce faible taux de scolarisation des tradipraticiens à Aguié, avec 38\% d'analphabètes, $14 \%$ des scolarisés, $7 \%$ d'alphabétisés et $41 \%$ ayant fréquentés l'école coranique seulement. 55 espèces médicinales ont été inventoriées, réparties en 26 familles. Ce nombre est inférieur à celui recensé par Hassane (2008) dans la réserve de la biosphère de W du Niger (201 espèces). Cette différence pourrait être due au fait que la flore de la réserve de la biosphère est plus diversifiée. Cet inventaire de 
plantes médicinales dans les deux communes du département de Dogondoutchi a fait ressortir une dominance des familles des FabaceaeCaesalpinoideae, des Fabaceae-Mimosoideae et des Combretaceae. Les plantes ligneuses comportent le plus grand nombre en pharmacopée traditionnelle. Ce résultat est identique à celui de Nana-Sanon (2005) qui confirme que près de $90 \%$ des espèces recensées sont ligneuses. Au niveau de la plante, la feuille est plus utilisée $(42,85 \%)$ pour les soins que les autres organes. Ce résultat corrobore ceux de Mehdioui et Kaouadji (2007) au Maroc, Fezan et al. (2008) en Côte d'Ivoire, Erinoso et Aworinde (2012) au Nigéria, Garba et al. (2019) et Abdou Habou et al., (2020) au Niger. Cette importance donnée à la feuille dans les usages thérapeutiques pourrait être expliquée par sa facilité d'accès ou par son efficacité. La décoction $(43,07 \%)$ est le mode de préparation le plus cité dans cette étude, ce qui corrobore les résultats de plusieurs auteurs (Mehdioui et Kahouadji 2007 ; Fezan et al., 2008 ; Issoufou, 2011 et Sangaré et al., 2012). L'administration se fait par plusieurs voies : la voie orale qui est la plus fréquente, la voie cutanée et la voie nasale. Ces résultats présentent une conformité avec celui d'Issoufou (2011) qui a cité en plus de ces voix d'administration, la voix anale. Selon les tradipraticiens, la récolte des

\section{CONCLUSION}

Cette étude a permis de comprendre davantage l'importance de la diversité végétale, les connaissances des populations dans la pharmacopée traditionnelle, notamment concernant les maladies traitées, les organes utilisés, les modes de préparation et les modes d'administration. Les populations gardent encore la tradition dans l'utilisation des plantes médicinales mais cela cause également des conséquences sur l'environnement compte tenu plantes se fait avec soin, mais malgré cela, la mauvaise utilisation des outils sur les individus végétaux peut engendrer la perte de nombreuses espèces dans le milieu. L'exploitation des écorces et des racines met en danger les espèces concernées en provoquant leur dépérissement, ce qui compromet leur survie, une idée également soutenue par Abdou et al. (2014). La plupart des personnes enquêtées $(85,71 \%)$ ont hérité les connaissances de leurs parents ce qui rapproche ce résultat de ceux de Erinoso et Aworinde (2012) au Nigéria et Hui et al. (2012) en Inde. A l'issue de ces entretiens, il ressort que certaines des espèces à vertus médicinales sont en régression suite à leur utilisation abusive dans les deux communes. L'étude a prouvé une redondance de certaines espèces qui, parfois sont déclarées comme disparues ou en voie de disparition comme par exemple Lannea microcarpa, Vitex doniana, Combretum nigricans, Ficus platyphylla. D'après les tradipraticiens, bon nombre des espèces utilisées ont disparu ou en voie de disparition suite aux actions anthropiques. Ce résultat montre que la perte des espèces est due non seulement aux effets naturels mais en grande partie aux actions anthropiques, ce qui confirme la nécessité d'une surveillance attentive des espèces protégées, des réserves naturelles et des forêts classées.

de l'exploitation abusive des plantes. Pour pallier cette dégradation de l'environnement, il est nécessaire de réimplanter les espèces disparues les plus utiles en pépinières et aussi dans les champs, promouvoir la domestication de certaines espèces, sensibiliser la population locale dans la gestion intégrée des ressources naturelles en vue de préserver la biodiversité et d'encourager la création des jardins botaniques.

\section{REFERENCES BIBLIOGRAPHIQUES}

Abdou Habou M.K., Rabiou H., Abdou L., Ibrahim Mamadou M., Mahamane A., 2020. Connaissances ethnobotaniques et importance socioculturelle de Balanites aegyptiaca (L.) Del. dans le Centre-Est du
Niger. Afrique SCIENCE 16(4) 239 252.

Abdou L., Dan Guimbo I., Larwanou L., Inoussa M.M., Mahamane M., 2014. Utilisation de Prosopis africana (G. et Perr.) 
Taub dans le sud du département d'Aguié au Niger : les différentes formes et leur importance. Int. J. Biol. Chem. Sci.. 8(3): 1065-1074, DOI : http://dx.doi.org/10.4314/ijbcs.v8i3.20

Badiaga M., 2011. Étude ethnobotanique, phytochimique et activités biologiques de Nauclea latifolia (smith)., une plante médicinale Africaine récoltée au Mali. Thèse de Doctorat, Université de Bamako. 137 p.

Baggnian I., Abdou L., Yameogo J.T., Moussa I., Adam T., 2018. Étude ethnobotanique des plantes médicinales vendues sur les marchés du centre ouest du Niger. Journal of Applied Biosciences, 132, 13392-13403.

Betti J.L. \& Lejoly J., 2000. Les plantes indiquées comme antihelminthiques en thérapie traditionnelle dans la réserve de biosphère du Dja (Cameroun). Revue Soma 4-16.

CUD (Commune urbaine de Dogondoutchi), 2017. Plan de développement communautaire.

Erinoso S.M. \& Aworinde D.O., 2012. Ethnobotanical survey of some medicinal plants used in traditional health care in Abeokuta areas of Ogoun State, Nigeria. African Journal of Pharmacy and Pharmacology, 6 (18), 1352-1362.

Fezan H., Tra B., Guy M., Kohue C., Clejesson H., 2008. Etude de quelques plantes thérapeutiques utilisées dans le traitement de l'hypertension artérielle et du diabète : deux maladies émergentes en Côte d'Ivoire. Science et Nature, 5 (1), 3948.

Garba A., Amani A., Abdou L., Mahamane A., 2019. Perceptions et usages socioéconomiques du tamarinier (Tamarindus indica L.) dans le Sud-Ouest du Niger : Implications pour une domestication et une conservation durable. Journal of Animal and Plant Sciences, 40 (2), 6584-6602.

Guedje N.M., Mouamfon M., Bigombe Logo P., Abega S.C, \& Lejoly J., 2008. Impact de la gestion socio-économique et technique des forêts communautaires à l'échelle des économies familiales : Cas de Kompia et Kabilone (Est-Cameroun). In : Roulet P.A., Assemaker P. (eds). Governance et Environnement en Afrique Centrale : le modèle participatif en question. Musée Royal de l'Afrique Centrale Tervuren Belgique, pp. 139157.

Hassane H., 2008 :Répertoire des espèces végétales les plus couramment utilisées en pharmacopée traditionnelle et impact des techniques de prélèvement sur la diversité biologique dans la réserve de Biosphère du W du Niger, MEMOIRE DE DEA GEOGRAPHIE, 99p.

Hui T., Kalita P., Dwivedi P., Das A.K., Nima D.N., 2012. Herbal medicines used in the treatmentof diabetes mellitus in Arunachal Himalaya, northeast, India. Journal of Ethnopharmacology, 141 (2012): 786-795.

INS (Institut national de la statistique), 2016. Monographie régionale de Dosso. 122p

Issoufou S., 2011. Etude ethnobotanique sur la présence et l'utilisation des plantes médicinales dans les marchés de Maradi, Rapport de stage, UAM, Faculté d'Agronomie, 49p.

Jiofack T., Fokunang C., Guedje N.M., Kemeuze V., Fongnzossie E., Nkongmeneck B.A, Mapongmetsem P.M, Tsabang N., 2010. Ethnobotanical uses of medicinals plants of two ethnoecological regions of Cameroon. International Journal of Medicine and Medical Sciences, 2 (3): 60-79.

Koné M.W., Atindehou K.K., Téré H., Traoré D., 2002. Quelques plantes médicinales utilisées en pédiatrie traditionnelle dans la région de Ferkessédougou (Côte d'Ivoire). Bioterre, $30:$ 30-36.

Mehdioui R. \& Kahouadji A., 2007. Etude ethnobotanique auprès de la population riveraine de la forêt d'Amsittène : cas de la commune d'Imi n'Tlit (Province d'Essaouira). Bulletin de l'Institut 
Scientifique, Rabat, section Sciences de la Vie, 29, 11-29.

Mike O.S., Emmanuel C.C., Olatunji M.S., Feyisola R.T., 2014. Ethnobotanical Survey of Plants Used in the Traditional Treatment of Female Infertility in Southwestern Nigeria. Ethnobotany Research and Applications, 12: 081-090.

Mpondo E.M., Didier S.D., Richard J.P., Alfred N., Christelle F.L.Y., 2012. Etude actuelle de la médecine traditionnelle dans le système de santé des populations rurales et urbaines de Douala (Cameroun). Journal of Applied Biosciences, 55, 4036- 4045.

Nana-Sanon P., 2005. Plantes médicinales utilisées pour les soins des enfants dans la commune de Ouagadougou : inventaire et culture de quelques espèces. Mémoire de fin d'études d'Ingénieur des Eaux et Forêts. 75p.

Pousset J.L., 1989. Plantes médicinales africaines - Utilisation pratique. Ellipses, ACCT : Paris ; 66-86.

R Development Core Team (2005). R: A language and environment for statistical computing. R Foundation for Statistical Computing, Vienna, Austria. ISBN 3900051-07-0, URL: http://www.Rproject.org.

Roufai D.A., 2013. Etude ethnobotanique et identification de quelques espèces locales prometteuses dans le complexe des forêts classées de Dan Kada Dodo-Dan Gado, département d'Aguié. Mémoire pour l'obtention du Diplôme de Master 2 en Biodiversité et Gestion de l'Environnement Soudanien et SahéloSaharien (BGESSS), 32 p.

Sangaré M.M., Sina H., Dougnon J., Babayala B., Ategbo JM., Dramane L.K., 2012. Etude ethnobotanique des plantes hépatotropes et l'usage traditionnel de Gomphrena celosioides Mart. (Amarenthaceae) au Bénin. International Journal of Biological and Chemical Sciences, 6 (6), 5008- 5021.
Togola A., Diallo D., Dembélé S., Barsett H., Smestad Paulsen B., 2005. Ethnopharmacological survey of different uses of seven medicinal plants from Mali, (West Africa) in the regions Doila, Kolokani and Siby. Journal of Ethnobiology and Ethnomedicine, 1:7. 